\title{
FELINE AMINOPEPTIDASE N IS A RECEPTOR FOR ALL GROUP I CORONAVIRUSES
}

\author{
Dina B. Tresnan and Kathryn V. Holmes \\ Department of Microbiology \\ University of Colorado Health Sciences Center \\ Denver, Colorado 80262
}

\begin{abstract}
Human coronavirus HCV-229E and porcine transmissible gastroenteritis virus (TGEV), both members of coronavirus group I, use aminopeptidase N (APN) as their cellular receptors. These viruses show marked species specificity in receptor utilization as they can only use APN of their respective species to initiate virus infection. Feline and canine coronaviruses are also group I coronaviruses. To determine whether feline APN could serve as a receptor for feline coronaviruses (FCoVs), we cloned the cDNA encoding feline APN (fAPN) by PCR from feline cells and stably expressed it in FCoV-resistant mouse or hamster cells. These became susceptible to infection with either of several biotypes of FCoVs. The expression of recombinant fAPN also made hamster and mouse cells susceptible to infection with other group I coronaviruses, including several canine coronavirus strains, transmissible gastroenteritis virus (TGEV), and human coronavirus HCV-229E. Thus, fAPN served as a functional receptor for each of these coronaviruses in group I. As expected, fAPN could not serve as a receptor for mouse hepatitis virus (MHV), a group II coronavirus which uses murine biliary glycoproteins as receptors. Thus, fAPN acts as a common receptor for coronaviruses in group I, in marked contrast to human and porcine APN glycoproteins which serve as receptors only for human and porcine coronaviruses, respectively. These observations suggest that cats could serve as a "mixing vessel" in which simultaneous infection with several group I coronaviruses could lead to recombination of viral genomes.
\end{abstract}

\section{INTRODUCTION}

Coronaviruses in group I cause disease in many different species of animals and humans, generally in a host specific manner (Wege, 1982). The members of group I include 
two feline coronaviruses (FCoVs), feline infectious peritonitis virus (FIPV) and feline enteric coronavirus $(\mathrm{FeCV})$; two porcine coronaviruses, transmissible gastroenteritis virus (TGEV) and porcine respiratory coronavirus (PRCV); canine coronavirus (CCV); and human coronavirus 229E (HCV-229E) (Holmes and Lai, 1996). Except for FIPV, which causes systemic disease, infections with these viruses are generally limited to the enteric or respiratory tracts.

The feline coronaviruses cause disease in domestic and exotic Felidae. Isolates of FIPV from diseased cats vary markedly in virulence in vivo and cytopathogenicity in vitro.

While some isolates called $\mathrm{FeCV}$ cause inapparent or mild infection in the enteric tract in kittens (Pedersen, 1987), other strains known as FIPV cause a chronic, systemic immune-mediated disease called feline infectious peritonitis (FIP) (Scott, 1986a). This disease is characterized by fibrinonecrotic and pyogranulomatous peritonitis and pleuritis and is usually fatal (Barlough and Stoddart, 1990; Pedersen and Boyle, 1980).

Based on serological studies, FCoVs have been subdivided into biotypes I and II (Pedersen et al., 1984). Type II FCoVs are antigenically more closely related to CCV than to type I strains and also more closely resemble CCV in growth in tissue culture (Pedersen et al., 1984). Comparative analysis of nucleotide sequences of feline and canine coronaviruses suggests that type II FCoVs may have arisen by RNA recombination between type I FCoV and CCV (Herrewegh, et al., 1995).

Although, in general, the group I coronaviruses can only infect cultured cells of their normal host species, feline cells in culture can be infected with CCV, TGEV and HCV-229E as well as the FCoVs (Levis et al., 1995). All of these viruses bind to brush border membranes isolated from cat intestines (Holmes and Compton, 1995). In addition, cats can be experimentally infected with non-feline coronaviruses in group I. The infected animals develop anti-viral antibody and may shed some virus, but they do not exhibit any clinical disease and infection does not protect them from subsequent infection with FCoVs (Scott, 1986b; Barlough et al., 1985; Barlough et al., 1984; Reynolds and Garwes, 1979).

Aminopeptidase N (APN) is a $150-\mathrm{kDa}$ plasma membrane-associated type II glycoprotein with metalloprotease activity. It is expressed on the plasma membranes of epithelial cells of the intestinal brush border, renal proximal tubules, and respiratory tract, the synaptic membranes of the central nervous system and cells of the granulocyte and monocyte lineages (Look et al., 1989; Kenny and Maroux, 1982). Cellular entry of both HCV-229E and the porcine coronaviruses TGEV and PRCV is mediated by the same APN receptor (Delmas et al., 1994a; 1992; Yeager et al., 1992). However, HCV-229E cannot utilize porcine APN (pAPN) as a receptor, and TGEV cannot utilize human APN (hAPN) as a receptor (Levis, et al., 1995; Delmas et al., 1994b; 1992). Hamster (BHK) cells transfected with pAPN were not susceptible to FIPV or CCV, suggesting that the pAPN expressed in these cells could not function as a receptor for these cat and dog coronaviruses (Delmas et al., 1994a). Thus, in these instances, receptor specificity appears to be a major determinant of the observed species specificity of HCV-229E and TGEV infection.

Since APNs serve as receptors for several group I coronaviruses, we investigated whether feline APN (fAPN) could serve as a receptor for FCoVs. Using primers that recognize consensus sequences in APN genes from other species, a cDNA encoding feline APN was amplified by PCR from feline cell cDNA, cloned, and then expressed in mouse or hamster cells. Cells transfected with the feline APN gene were challenged with FCoVs and other group I coronaviruses to determine whether fAPN was a functional receptor for these viruses. 


\section{MATERIALS AND METHODS}

Methods were as described in Tresnan et al., 1996. Feline coronavirus strain DF-2 was obtained from American Type Culture Collection (ATCC) (Gaithersburg, MD). Canine coronavirus strain TN-449 was obtained from ATCC, and CCV/K378 was kindly provided by Leland E. Carmichael (James A. Baker Institute, Cornell University, Ithaca, NY). To increase the level of expression of recombinant fAPN, a new construct called fAPN-K was engineered using PCR. This construct contained an improved Kozak consensus sequence (Kozak, 1987) in comparison with the original fAPN construct (Tresnan, et al., 1996). Hamster or mouse cells transfected with fAPN-K became susceptible to infection with feline coronaviruses at a higher rate than cells transfected with the original fAPN construct.

\section{RESULTS}

Our approach to studying the virus receptor activity and species specificity of fAPN was to clone and express a full-length cDNA clone of fAPN in FCoV-resistant cells, and then test them for susceptibility to coronavirus infection. The complete nucleotide sequence of fAPN cDNA was determined from a full length cDNA and three independently cloned overlapping segments of the APN cDNA (Tresnan et al., 1996). Pairwise alignment of the amino acid sequence of fAPN with the sequences of hAPN or pAPN revealed 78 and $77 \%$ identity, respectively, using the human (M22324) and porcine (Z29522 ) APN sequences obtained from the GenBank database.

To determine whether the fAPN glycoprotein is a functional receptor for FCoVs, the full length cDNA encoding fAPN was stably expressed in hamster (BHK) or mouse (NIH3T3) cell lines, which are normally resistant to FCoV infection. The stably transfected cells were inoculated with either of several strains of FIPV including 79-1146, UCD-1, and DF-2, or mock infected with medium. Antigens of all three strains of FIPV were detected in the cytoplasm of the fAPN-transfected mouse or hamster cells by 6 hours post inoculation (p.i.), but no viral antigens were detected in hamster or mouse cells stably transfected with the vector alone and then inoculated with FIPV (Table 1 and 2; Tresnan et al., 1996). In hamster cells transfected with the fAPN-K construct, which contained the improved Kozak consensus sequence, and inoculated with FIPV 79-1146, a higher per cent of the cells was infected and there was more extensive fusion than in cells transfected with the original

Table 1. Feline coronavirus strains that can utilize fAPN as a receptor

\begin{tabular}{lcccc}
\hline Strain & Biotype $^{\text {a }}$ & $\begin{array}{c}\text { Growth in } \\
\text { cell culture }\end{array}$ & Disease & $\begin{array}{c}\text { Use fAPN as } \\
\text { a receptor }^{\text {b }}\end{array}$ \\
\hline FIPV & & & & \\
$79-1146$ & II & + & FIP & + \\
DF-2 & II & + & FIP & + \\
UCD-1 & I & poor & FIP & + \\
FeCV & & & & + \\
$79-1683$ & II & + & Enteritis & + \\
\hline
\end{tabular}

${ }^{a}$ Biotypes of different strains were reviewed by Herrewegh et al., 1995.

${ }^{b}$ The ability to use fAPN as a receptor was demonstrated by the production of viral antigens in the cytoplasm of hamster and or mouse cells transfected with fAPN after inoculation with each strain of FCoV (Tresnan et al., 1996). 
fAPN construct (Fig. 1). Hamster and mouse cells transfected with fAPN or with vector alone were also inoculated with $\mathrm{FeCV}$, an enterotropic feline coronavirus closely related to FIPV. FeCV viral antigens were detected in the cytoplasm of fAPN-transfected NIH3T3 cells and BHK, but not in cells transfected with vector alone. Thus, fAPN serves as a receptor for all feline coronaviruses tested, including several biologically different strains of FIPV and one of FeCV. Expression of recombinant fAPN in mouse or hamster cells is sufficient to allow FCoV binding and entry and the synthesis of viral antigens.

To determine whether fAPN-transfected hamster cells could produce infectious FIPV or FeCV, these cells were inoculated with FIPV 79-1146 or FeCV 79-1683, and the infectious virus released into the supernatant medium was titered by plaquing on Fcwf cells. With both FCoVs, infectious virions were produced and released into the medium from fAPN-transfected BHK cells, but not from BHK cells transfected with vector alone (data not shown).

Because the Fcwf line of feline cells is susceptible to infection by other coronaviruses in group I including CCV, TGEV and HCV-229E in addition to FCoVs (Table 2 and Levis et al., 1995), we tested these viruses for infectivity on fAPN-transfected BHK and fAPN-transfected NIH3T3 cells. The fAPN-transfected BHK and NIH3T3 cells or control cells transfected with vector alone were inoculated with HCV-229E, TGEV, or with MHV-A59 and with several strains of CCV including 1-71, K378 and TN-449. Transfected cells were infected in two separate experiments and multiple cover slips were analyzed to determine the percentage of infected cells by immunolabeling with anti-viral antibody. The fAPN-transfected hamster and mouse cells were susceptible to infection
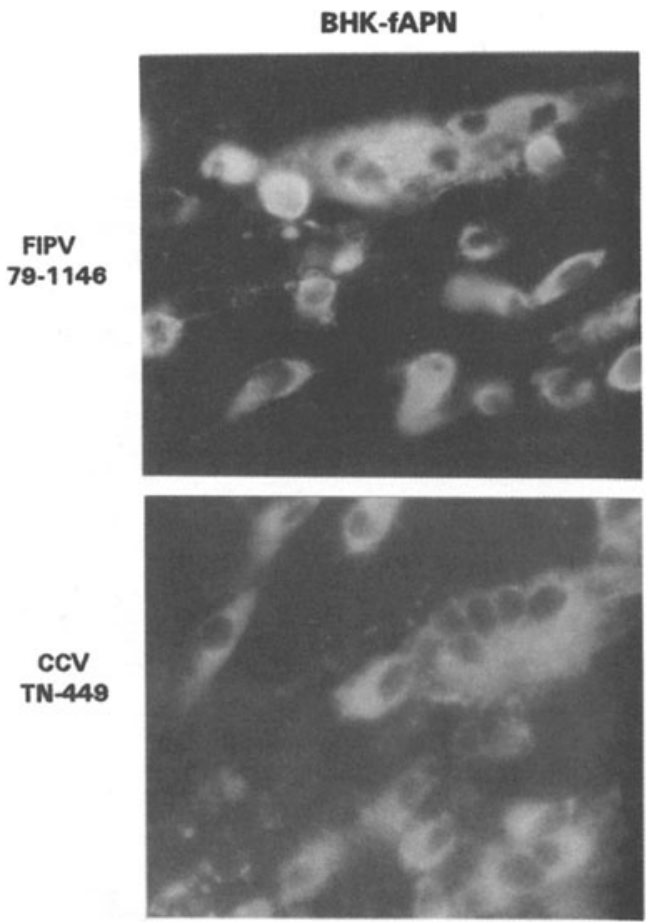

Figure 1. Recombinant feline aminopeptidase $\mathrm{N}$ can serve as a receptor for both feline and canine coronaviruses. Hamster cells transfected with feline aminopeptidase $\mathrm{N}$ were inoculated with feline coronavirus FIPV $79-1146$ or canine coronavirus TN449. Viral antigens were detected in infected cells by immunofluorescent labeling. 
Table 2. Receptor activity of fAPN for various coronaviruses

\begin{tabular}{lcccccc}
\hline & FIPV & FeCV & CCV & & \\
& $79-1146$ & $79-1683$ & $1-71$ & TGEV & HCV-229E & MHV-A59 \\
\hline Fcwf & $++^{\mathrm{a}}$ & + & + & + & + & - \\
BHK-pCR3 & - & - & - & - & - & - \\
BHK-fAPN & + & + & + & + & + & - \\
\hline a Susceptibility to coronavirus infection was determined by the detection of viral antigens in in- \\
fected cells using an indirect immunofluorescence assay (Tresnan et al., 1996).
\end{tabular}

with each of the coronaviruses in group I as shown by viral antigen production in the cytoplasm of some of the transfected cells (Table 2; Fig.1, and Tresnan et al., 1996). Neither mouse or hamster cells transfected with vector alone was infected by any of the group I coronaviruses tested. Thus, fAPN served as a receptor for CCV, HCV-229E and TGEV as well as for FCoVs. Because these experiments were done using pools of cells stably transfected with fAPN, we plan further studies on cloned cell lines expressing known levels of fAPN in order to determine whether fAPN is a more efficient receptor for feline coronaviruses than for other group I coronaviruses.

The fAPN-transfected BHK cells were not susceptible to infection with murine coronavirus MHV-A59. As expected, both NIH3T3 cells transfected with vector alone (data not shown) and fAPN-transfected NIH3T3 cells were susceptible to MHV infection because these mouse cells express the biliary glycoproteins that serve as receptors for MHV (Holmes and Dveksler, 1994).

\section{DISCUSSION}

We have shown that fAPN serves as a receptor for strains of FCoVs that differ markedly in their ability to grow in cultured feline cells and in their virulence in cats. In addition, we found that fAPN can serve as a receptor for all of the non-feline coronaviruses in group I that were tested (Tresnan, et al., 1996). The common use of APN as a receptor for related coronaviruses that infect different species has important implications for the evolution of this group of coronaviruses. The phylogenetic relationships between group I coronaviruses were examined by comparing the nucleotide sequences of their spike protein genes. This work showed that canine coronaviruses are more similar to FCoVs than to the porcine coronaviruses, while the spike protein sequences of HCV-229E clearly place that virus in a separate cluster (Wesseling, et al., 1994). The close relationships among these related coronaviruses and their use of a common molecule as a receptor suggests that they may have evolved from a common ancestor. Perhaps FCoV was the first ancestral group I coronavirus that exploited APN as a receptor. The virus could then have adapted to different species by a series of mutations in the $\mathrm{S}$ gene that optimized the usage of species-specific determinants on their APN receptors as shown for both porcine and human APN (Delmas et al. 1994a). Mutations in the spike protein that could optimize adaptation to species specific APN receptors may include altering the receptor binding site of S, its ability to undergo a conformational change, and/or its ability to mediate fusion of the viral envelope with the host membranes during virus entry.

Although APN molecules from various species have been shown to function as receptors for some group I coronaviruses, the specific functional domains that bind to the re- 
ceptor glycoprotein have not yet been completely defined. Using chimeras between pAPN and hAPN, Delmas and co-workers identified a region on pAPN between amino acids 717 and 813 , located 330 amino acids downstream from the active site of the enzyme, that determined its ability to serve as a receptor for TGEV (Delmas et al., 1994a). Recently, a chimera containing amino acids 643 to 841 from canine APN in the framework of human APN was shown to act as a receptor for CCV, FIPV and TGEV (Benbacer et al., 1997). The amino acid sequences of the APN glycoproteins of different species differ markedly in this region. Unexpectedly, using these same chimeras, the corresponding region of hAPN was not responsible for the species specificity of HCV-229E infection. Instead, a small region containing amino acids 260-353 of human APN was shown to determine the species specificity of HCV-229E infection (Kolb et al., 1996). Although chimeric APN glycoproteins help to define domains that affect the species specificity of group I coronavirus receptors, they do not define the precise areas of interaction of the $\mathrm{S}$ glycoproteins with the receptors. Some contributions to the functions of the receptor glycoprotein may be provided by the framework of the APN glycoprotein into which a segment from another species is inserted, in addition to the functions provided by the inserted segment.

We have shown that the full length fAPN glycoprotein can serve as a common receptor for all of the coronaviruses in group I. This is in marked contrast to the porcine and human APN glycoproteins which function as receptors only for coronaviruses that cause disease in a single species (Kolb et al.,1996; Delmas, et al.1994a). The non-feline coronaviruses in group I can infect cats without causing disease (Barlough et al., 1985; 1984). These viruses probably infect cats in vivo by using feline APN as a receptor, as they can do in vitro (Tresnan, et al. 1996). If all group I coronaviruses can infect cat cells in vivo using their common fAPN receptor, then it is possible that recombination between different coronaviruses in group I could occasionally occur in a cell of a cat that is simultaneously infected with several of these viruses. Thus, the cat could serve as a "mixing vessel," allowing recombination and shuffling of genes from different group I coronaviruses. Strong support for this hypothesis is provided by the observation that type I FCoVs may have arisen naturally by recombination between a type II FCoV and CCV (Herrewegh, et al., 1995). While most recombinant coronaviruses might be poorly suited for transmission from animal to animal, rarely a recombinant virus might arise that can replicate to high titers in vivo and be transmitted efficiently. Such a transmissible recombinant coronavirus would be a candidate for causing an emerging virus disease either in cats or another host species.

\section{ACKNOWLEDGMENTS}

We are grateful to the excellent technical assistance of John Schneider and James Ahn and to Bruce Zelus, David Wentworth and Dianna Blau for thoughtful comments on the manuscript. We also are grateful to the many colleagues who provided us with virus strains and cell lines. This work was supported by U.S. Public Health Service grant AI26075 and by Physician Scientist Award K11 AI01151 to D.B.T.

\section{REFERENCES}

Barlough, J. E., and Stoddart, C. A., 1990, Feline coronaviral infections, in: Infectious diseases of the dog and cat, (C. E. Greene, ed.), W. B. Saunders Co., Philadelphia, pp. 300-312.

Barlough, J. E., Stoddart, C. A., Sorresso, G. P., Scott F. W., and Jacobson, R. H.,1984, Experimental inoculation of cats with canine coronavirus and subsequent challenge with feline infectious peritonitis virus, Lab. Anim. Sci. 34: 592-597. 
Barlough, J. E., Johnson-Lussenburg, C. M., Stoddart, C. A., Scott, F. W., and Jacobson, R. H., 1985, Experimental inoculation of cats with human coronavirus $229 \mathrm{E}$ and subsequent challenge with feline infectious peritonitis virus, Can. J. Comp. Med. 49: 303-307.

Benbacer, L., Kut, E., Besnardeau, L., Laude, H., and Delmas, B., 1997, Interspecies aminopeptidase-N chimeras reveal species specific receptor recognition by CCV, FIPV, and TGEV, J. Virol. 71: 734-737.

Delmas, B., Gelfi, J., L'Haridon, R., Vogel, L. K., Sjöström, H., Norén, O., and Laude, H., 1992, Aminopeptidase $\mathrm{N}$ is a major receptor for the enteropathogenic coronavirus TGEV, Nature (London) 357: 417-419.

Delmas, B., Gelfi, J., Kut, E., Sjöström, H., Norén, O., and Laude, H., 1994a, Determinants essential for the transmissible gastroenteritis virus-receptor interaction reside within a domain of aminopeptidase $\mathrm{N}$ that is distinct from the enzymatic site, J. Virol. 68: 5216-5224.

Delmas, B., Gelfi, J., Sjöström, H., Norén, O., and Laude, H., 1994b, Further characterization of aminopeptidase $\mathrm{N}$ as a receptor for coronaviruses, $A d v$. Exp. Med. Biol. 342: 293-298.

Herrewegh, A. A. P. M., Vennema, H., Horzinek, M.C., Rottier, P.J.M., and de Groot, R.J., 1995, The molecular genetics of Feline coronaviruses: Comparative sequence analysis of the ORF7a/7b transcription unit of different biotypes, Virology 212: 622-631.

Holmes, K.V., and Dveksler, G.S., 1994, Specificity of coronavirus/receptor interactions, in: Cellular Receptors for Animal Viruses, (E. Wimmer, ed.), Cold Spring Harbor Laboratory Press, pp. 403-443.

Holmes, K. V., and Compton, S. R., 1995, Coronavirus receptors, in: The Coronaviruses, (S. Siddell, ed.), Plenum Press, New York, pp. 55-72.

Holmes, K. V., and Lai, M. M. C., 1996, Coronaviridae: The viruses and their replication, in: Virology (Fields), 3rd ed., (B. N. Fields, D. M. Knipe and P. M. Howley, eds.), Lippincott-Raven Publishers, Philadelphia, pp. $1075-1093$.

Kenny, A. J., and Maroux, S., 1982, Topology of microvillar membrane hydrolases of kidney and intestine, Physiol. Rev. 62: 91-128.

Kolb, A., Maile, J., Heister, A., and Siddell, S., 1996, Characterization of functional domains in the human coronavirus 229E receptor, J. Gen. Virol. 77: 2515-2521.

Kozak, M., 1987, An analysis of 5 -noncoding sequences from 699 vertebrate messenger RNAs, Nucleic Acids Res. 15:8125-8148.

Levis, R., Cardellichio, C.B., Scanga, C.A., Compton, S.R., and Holmes, K.V., 1995, Multiple receptor-dependent steps determine the species specificity of HCV-229E infection, Adv. Exp. Med. Biol. 380: 337-344.

Look, A. T., Ashmun, R. A., Shapiro, L. H., and Peiper, S. C., 1989, Human myeloid plasma membrane glycoprotein CD13 (gp150) is identical to aminopeptidase N, J. Clin. Invest. 83: 1299-1307.

Pedersen, N. C., 1987, Virologic and immunologic aspects of feline infectious peritonitis virus infection, Adv. Exp. Med. Biol. 218: 529-550.

Pedersen, N. C., and Boyle, J. F., 1980, Immunologic phenomena in the effusive form of feline infectious peritonitis, Am. J. Vet. Res. 41: 868-876.

Pedersen, N.C., Black, J.W., Boyle, J.F., Evermann, J.F., McKeirnan, A.J., and Ott, R.L., 1984, Pathogenic differences between various feline coronavirus isolates, Adv. Exp. Med. Biol. 173 :337-344.

Reynolds, D. J., and Garwes D. J., 1979, Virus isolation and serum antibody responses after infection of cats with transmissible gastroenteritis virus, Arch. Virol. 60:161-166.

Scott, F. W., 1986a, Feline infectious peritonitis and other feline coronaviruses, in: Current veterinary therapy IX, ( R. W. Kirk, ed.), W. B. Saunders Co., Philadelphia, pp. 1059-1062.

Scott, F. W., 1986b, Immunization against feline coronaviruses, Adv. Exp. Med. Biol. 218:569-576.

Tresnan, D.B., Levis, R., and Holmes, K.V., 1996, Feline aminopeptidase N (fAPN) serves as a receptor for feline, canine, porcine and human coronaviruses in serogroup I, J. Virol. 70:8669-8674

Wege, H., Siddell, S., and ter Meulen, V., 1982, The Biology and Pathogenesis of Coronaviruses, Curr. Topics Microbiol. Immunol. 99: 165-200.

Wesseling, J.G., Vennema, H., Godeke, G.J., Horzinek, M.C., Rottier, P.J., 1994, Nucleotide sequence and expression of the spike (S) gene of canine coronavirus and comparison with the $\mathrm{S}$ proteins of feline and porcine coronaviruses, J. Gen. Virol. 75: 1789-1794.

Yeager, C. L., Ashmun, R. A., Williams, R. K., Cardellichio, C. B., Shapiro, L. H., Look, A.T., and Holmes, K. V., 1992, Human aminopeptidase $\mathrm{N}$ is a receptor for human coronavirus 229E, Nature (London) 357: $420-422$. 